\title{
La méthode liste. Textualité et créativité
}

\section{Rudolf Mahrer}

\section{OpenEdition}

\section{Journals}

Édition électronique

URL : http://journals.openedition.org/genesis/3353

DOI : 10.4000/genesis.3353

ISSN : 2268-1590

\section{Éditeur :}

Presses universitaires de Paris Sorbonne (PUPS), Société internationale de génétique artistique littéraire et scientifique (SIGALES)

\section{Édition imprimée}

Date de publication : 15 décembre 2018

Pagination : 13-33

ISBN : 979-10-231-06282

ISSN : $1167-5101$

\section{Référence électronique}

Rudolf Mahrer, "La méthode liste. Textualité et créativité », Genesis [En ligne], 47 | 2018, mis en ligne le 15 décembre 2019, consulté le 21 janvier 2021. URL : http://journals.openedition.org/genesis/3353 DOI : https://doi.org/10.4000/genesis.3353 


\section{La méthode liste. Textualité et créativité}

Rudolf Mahrer

E

n vue d'accomplir une tâche complexe, comme par exemple l'écriture d'un texte, il arrive qu'on fasse des listes. Pourquoi ? Où réside le pouvoir heuristique de la liste? Comment rendre compte de sa présence régulière dans les dossiers de genèse ? Pour répondre à ces questions, je propose d'envisager la liste sous un angle linguistique : comme une pratique discursive qui met en action un ensemble de propriétés langagières selon une organisation caractéristique et en vue d'une finalité donnée. Ce que j'aimerais préciser autrement dit, ce sont les propriétés textuelles qui expliquent les vertus créatives de la liste 1 .

La question de l'activité d'écriture n'a guère été posée en termes de textualité : pensés au filtre de la notion d'avant-texte, les témoins de la production écrite ne font pas (encore) texte. Philippe Hamon relaie et adopte ce point de vue lorsqu'il écrit, à propos des ébauches de Zola ou des carnets intimes de Baudelaire : «La liste y est un non-texte en attente de devenir texte, une série de consignes en attente de devenir signes 2 .» Voilà qui incline peu à interroger l'organisation textuelle de la liste. Pour moi, la capacité de la liste à stimuler l'invention est à chercher dans son organisation linguistique locale et globale. En un mot, dans sa textualité

Ma position s'appuie sur l'observation suivante : parmi les documents génétiques, à côté de documents préparatoires qui épousent la forme générique du texte préparé (des essais d'articles, des débuts de contes, des vers isolés à assembler en poèmes...), il existe des pratiques qui adoptent une organisation textuelle spécifique, différente de celle du genre préparé : le plan, le scénario ou encore la liste. Quand le romancier écrit un scénario ou un plan, il ne s'essaie pas encore à son roman : il recourt à des formes d'organisation, des moyens linguistiques et cognitifs, bref à des genres de textes spécifiquement préparatoires 3 .

Pour déterminer les propriétés qui font de la liste une activité propice à l'invention, je proposerai dans un premier temps une définition «praxéologique» de la liste (ce à quoi elle sert et comment elle le fait). Puis, sur la base de quelques exemples choisis parmi une cinquantaine de listes étudiées dans les archives du Centre de recherches sur les lettres

1. Philippe Hamon, spécialiste de listes aussi bien que de genèses, observe la récurrence des listes dans les dossiers préparatoires (Puisque réalisme il y a, Genève, La Baconnière, 2015, p. 23); Lucia Manea également, dans un article qui est, à ma connaissance, le premier à se pencher spécifiquement sur la liste en contexte d'écriture : «L'effet générateur de fiction de la liste : variations des carnets de composition au roman », dans S. Milcent-Lawson, M. Lecolle et R. Michel (dir.), Liste et effet liste en littérature, Paris, Classiques Garnier, coll. «Rencontres», 2013, p. 97-109.

2. Philippe Hamon, op. cit., p. 163.

3. Sur ce programme de recherche, visant à décrire la généricité et la textualité des documents génétiques, voir Rudolf Mahrer et Valentine Nicollier Saraillon, «Les brouillons font-ils texte? Le cas des plans pré-rédactionnels de C. F. Ramuz», dans J.-M. Adam (dir.), Faire texte. Frontières textuelles et opérations de textualisation, Besançon, Annales littéraires de l'université de Franche-Comté, 2015, p. 223-305. 


\section{La liste comme trace de l'activité énumérative}

romandes et de la Bibliothèque cantonale et universitaire de Lausanne ${ }^{4}$, j'énumérerai les propriétés de la liste en contexte de production écrite. Dans un troisième et dernier temps, j’esquisserai une typologie fonctionnelle des listes génétiques.

Aborder en linguiste le processus créatif, c'est se pencher sur les discours produits en cours de création pour déterminer de quelles activités ces documents sont les traces et pour comprendre en quoi ces activités et leurs traces sont des ressources pour l'invention ${ }^{5}$. On en jugera en articulant hypothèses praxéologiques sur les visées de l'activité manifestée par la liste et descriptions linguistiques de la liste comme forme textuelle.

En accord avec le métalangage spontané, admettons d'abord d'appeler énumération l'opération dont la liste résulte et réservons le terme de liste pour la réalisation graphique de cette opération 6 . On considérera donc ici - comme le fait Goody - que la liste est un objet écrit. Voilà bien sûr qui n'empêche pas qu'on effectue régulièrement, à l'oral, l'activité énumérative; il existe même un «intonème» codant spécifiquement l'énumération ouverte. Celle-ci est fondamentale au point d'avoir abouti à un signe du système prosodique 7 .

Mais en quoi consiste l'activité énumérative ? Admettons que le discours consiste à représenter des objets (ainsi que des sujets). Dans le fil de l'énonciation, le locuteur élabore ces objets, par le jeu d'opérations successives et hiérarchisées, et les organise en objets complexes, ou classe-objet ${ }^{8}:$ l'énumération est une manière spécifique de construire un tel objet complexe. En cela, elle s'apparente à la description. Par exemple, dans l'extrait de conte suivant :

J'ai contemplé, ce soir, une joyeuse assemblée d'enfants réunis autour de ce charmant jouet venu d'Allemagne qu'est un arbre de Noël. L'arbre était planté au milieu d'une grande table ronde et

\footnotetext{
4. J'ai grand plaisir à remercier les collègues qui m’ont guidé dans les archives : Stéphane Pétermann pour les fonds Guy de Pourtalès et Monique Saint-Hélier, Alessio Christen et Bruno Pellegrini pour le fonds Gustave Roud. 5. C'est par cette attention accordée à la matérialité et à la structure linguistique des documents qui accompagnent et stimulent la création que le généticien linguiste peut apporter une contribution complémentaire à la psychologie de la créativité. Les psychologues élaborent leurs modèles essentiellement à partir du discours «métacréatif» des agents et à partir de protocoles expérimentaux qui prennent peu en considération la forme linguistique des opérations cognitives décrites. Pour un panorama, voir Todd Lubart, Christophe Mouchiroud, Sylvie Tordjman, Frank Zenasni, Psychologie de la créativité, Paris, Armand Colin, 2015 (2e édition).

6. La définition du $T L F$ paraît indifférente à l'opposition oral/écrit, jusqu'au moment de faire référence à la disposition : «Suite continue, hiérarchisée ou non, de noms (de personnes ou d'objets) ou de signes généralement présentés en colonne.» Précision finale qui nous rappelle pourquoi on «dresse» des listes.

7. L'intonème liste se caractérise par un «ton bas sur la pénultième, syllabe finale très allongée, à contour montant puis légèrement descendant» (Groupe de Fribourg, Grammaire de la période, Berne, Peter Lang, 2012, p. 101). Pensez à votre manière d'énumérer le contenu de votre panier à commissions. Selon le Groupe de Fribourg, l'instruction prosodique en question «s'interprète le plus souvent comme une opération logicoïde de conjonction ou d'addition d'éléments, à laquelle s'ajoute, semble-t-il, quelque chose comme l'information < je sélectionne ces éléments au hasard dans un paradigme; je pourrais aussi bien en prendre d'autres $>$, qui produit un effet d'énumération ouverte» (p. 101-102). L'intonème décrit ne correspond donc qu'à un type particulier d'énumération.

8. La logique naturelle - «c'est-à-dire une logique qui se préoccupe des procédés de pensée qui permettent d'élaborer des contenus et de les relier les uns aux autres » (Jean-Blaise Grize, Logique naturelle, Presses universitaires de France, 1996, p. 80) - appelle en effet ces objets complexes «classe-objet».
} 
se dressait bien haut au-dessus de leurs têtes. Il était brillamment illuminé par une multitude de petites bougies...9

«Un arbre de Noël», «ce charmant jouet venu d'Allemagne», «planté au milieu d'une grande table ronde», «se dressait bien haut au-dessus de leurs têtes », «était brillamment illuminé par...» sont des expressions qui, selon différentes combinaisons syntaxiques et différentes opérations sémantiques, enrichissent la représentation de l'objet complexe « arbre de Noël». L'énumération, quant à elle, est une routine de représentation spécifique; on la trouve illustrée par la suite immédiate du conte de Dickens :

... et sur toutes ses faces il était couvert d'objets brillants qui étincelaient et jetaient mille feux.

Il y avait des poupées aux joues roses, dissimulées derrière les feuilles vertes; il y avait de vraies montres (ou du moins des montres pourvues d'aiguilles mobiles et susceptibles d'être remontées à l'infini) accrochées à d'innombrables rameaux; il y avait des tables vernies au tampon, ainsi que des chaises, des lits, des armoires, des horloges et divers autres meubles familiers (merveilleusement fabriqués en fer-blanc, à Wolverhampton) perchées au milieu des branches, comme pour préparer quelque féerique emménagement; [neuf "il y avait" plus tard...] bref, comme le déclarait avec ravissement devant moi, à voix basse, une jolie petite fille : «Il y avait de tout, et même davantage.»

La routine énumérative consiste à enrichir la représentation d'un objet (ici des «objets brillants ») en lui associant des éléments de même statut (ici des occurrences de la classe). L'énumération n'est pas restreinte à la relation être une occurrence de $X$, il peut s'agir aussi d'être une partie de $X$, une propriété de $X$, un aspect de $X$, un lieu où l'on rencontre $X . .$. ce qui la caractérise, c'est que tous les éléments représentant l'objet complexe entretiennent avec lui la même relation. L'itération stricte de la modalité d'insertion des éléments enrichissant la représentation de l'objet (ici, par juxtaposition des présentatifs «il y avait») produit souvent, comme ici, le sentiment d'une abondance sans fin.

Son fonctionnement rend l'activité énumérative particulièrement utile pour représenter des objets dont il est difficile de décrire l'identité par ancrage notionnel : le parcours des occurrences de la classe vaut alors pour meilleure représentation possible. Sa logique est extensionnelle. On peut énumérer la classe «des mots qui ressemblent à ombre», mais on ne peut guère la décrire mieux que par son nom.

En résumé, si la description représente un objet complexe en effectuant des opérations diverses, l'énumération est un rituel de représentation beaucoup plus contraint :

- Les éléments (propriétés ou objets) représentant l'objet complexe sont coordonnés entre eux : ils se situent donc tous à un même niveau (principe de l'homologie des conjoints).

- Les éléments énumérés sont des congénères reliés par la même relation syntaxique ou textuelle à leur contexte.

- L'homologie des éléments introduits pour représenter l'objet complexe s'étend au plan du contenu de l'expression : pour reconnaître une séquence textuelle comme une énumération, il faut reconnaître la contribution commune qu'apporte chaque élément à la représentation de l'objet complexe.

9. Charles Dickens, «Un arbre de Noël», traduction de Sylvère Monod, La Maison d'Âpre-Vent. Récits pour Noël et autres, Paris, Gallimard, «Bibliothèque de la Pléiade», 1979. 
Discutons le dernier point. Il faut distinguer l'objet complexe représenté par l'activité énumérative - ce qu'on appelle, après Hamon, le pantonyme - et la relation commune qu'entretiennent les éléments avec cet objet. Canne, moustache, chapeau melon est une liste dont le pantonyme pourrait être «Charlot», mais qui n'est pas «une liste de Charlot», ni de ses parties, ni même de ses accessoires. Si c'est une liste, c'est celle de ses attributs. Derrière les relations variables (partie de, accessoire de, propriété de...), repérer une séquence comme le produit d'une énumération suppose de reconnaître la relation commune qui lie tous les éléments à l'objet ${ }^{10}$.

Sur le plan cognitif, l'opération énumérative suppose ainsi la synergie de deux activités : un geste de synthèse regroupant une pluralité d'éléments en une classe-objet commune et un geste analytique déclinant la classe en éléments. L'ancrage notionnel réalisé par un titrepantonyme envisage l'énumération sous un angle synthétique, alors qu'un titre dénotant la relation commune l'appréhende de manière plus analytique. On verra que dans la dynamique de la création, les scripteurs qui listent vont parfois de l'idée du tout à celle des parties, parfois de la pluralité à l'unité intégrante.

La définition proposée doit permettre d'expliquer pourquoi plus les éléments d'une série sont hétérogènes, plus celle-ci est difficile à interpréter en tant qu'énumération. Ajoutons encore chariot à canne, moustache, chapeau, et l'on aboutit à quelque chose comme... une liste de mots associables à Charlot (selon des opérations portant aussi bien sur le contenu du mot que sur son expression). L'énumération de l'hétérogène se replie du monde vers le langage. Dans la fameuse «encyclopédie chinoise» de Borges 11 , c'est l'impossibilité de dégager la relation commune des éléments à une classe qui rend la liste impensable : «L'absurde ruine le et de l'énumération en frappant d'impossibilité le en où se répartiraient les choses énumérées » écrit Foucault - à moins bien sûr de considérer ce lieu comme celui du langage et de penser la liste comme énumération de mots. Mais cette parade interprétative est bloquée par le texte de Borges qui désigne la relation commune des éléments à l'objet complexe («les animaux ») comme étant ses divisions, nous forçant ainsi, «dans l'impossibilité nue de penser cela», à reconsidérer nos procédés de catégorisation et donc d'organisation du monde.

\section{Caractérisation textuelle de la liste}

Les listes présentent régulièrement trois propriétés textuelles qui sont relatives à la mise en facteur commun que réalise l'activité énumérative.

La plus évidente de ces propriétés est topographique : la verticalisation des listes signale ce qu'on appelle souvent le «déploiement d'un paradigme», c'est-à-dire le fait que l'avancée sur l'axe syntagmatique décline les items d'une même classe. À l'oral, l'imposition du même

10. De manière analogue, si l'on ajoute quelques noms de légumes à une liste de fruits, qu'obtient-on? Une liste de fruits-et-légumes. Certains informateurs soumis à cette question répondent : «Une liste de fruits et une liste de légumes. » Ils refusent de convoquer la classe «fruits et légumes » (dont le pantonyme est pourtant un syntagme figé). Ils confirment à leur manière que la reconnaissance d'une énumération suppose le repérage de la relation commune qui lie les éléments à la classe (ici, être une occurrence de).

11. On ne se lasse pas de la citer : «les animaux se divisent en : a) appartenant à l'Empereur, b) embaumés, c) apprivoisés, d) cochons de lait, e) sirènes, f) fabuleux, g) chiens en liberté, h) inclus dans la présente classification, i) qui s'agitent comme des fous, j) innombrables, $\mathrm{k}$ ) dessinés avec un pinceau très fin en poils de chameau, 1) et caetera, m) qui viennent de casser la cruche, n) qui de loin semblent des mouches » (selon Foucault au tout début des Mots et les Choses, Gallimard, 1966). 
contour intonatif sur les constituants intégrés dans une série énumérative ressortit à une logique iconique analogue à celle du retour à la ligne : chaque constituant segmental frappé de la même propriété suprasegmentale apporte une même contribution à la classe représentée.

Concernant la texture ensuite (régularités textuelles situées sur le plan de l'expression), les éléments mis en série présentent habituellement de forts parallélismes morphosyntaxiques, qui viennent signaler, stylistiquement si l'on veut, l'identité fonctionnelle des éléments énumérés et l'identité de leur relation à la classe qu'ils constituent (dans l'exemple de Dickens : «il y avait» + syntagme nominal indéfini pluriel + extensions).

Un mot enfin de la progression qui caractérise les séquences énumératives. Sans doute en raison de la modalité d'insertion, unique et répétée, des éléments dans la classe, l'énumération s'est spécialisée comme une activité cognitivo-discursive sans plan nécessaire. D'où cette observation, un peu simpliste, selon laquelle la liste n'aurait pas d'ordre. Certes, chaque liste est «rappelée à l'ordre» par la linéarité de l'énonciation. En outre, pour limiter l'arbitraire, guider la progression ou rassurer le lecteur, les énumérations peuvent s'appuyer sur des programmes textuels (bas-haut, gauche-droite, points cardinaux, ordre alphabétique, etc.). Ces marqueurs et leur organisation ne sont néanmoins pas définitoires de la pratique énumérative et si l'on permute les éléments d'une liste, on obtient toujours une liste.

La possible absence de programme, caractéristique de l'énumération comme type d'activité cognitivo-discursive, n'empêche ni le scripteur, ni l'interprète d'assigner une raison à l'énumération (au sens de la raison des suites mathématiques). Il est vraisemblable pourtant que moins l'on identifiera de logique à l'ordre de la liste, plus on aura le sentiment d'avoir à faire à une «pure énumération».

En résumé, on définira l'énumération comme une activité discursive consistant à représenter une classe-objet en nommant tout ou partie de ses éléments. Cette activité est à la fois manifestée et structurée par un texte ou une séquence textuelle dont le plan est libre, qui ne représente qu'une seule classe-objet et dont les éléments, non hiérarchisés, entretiennent avec l'objet complexe représenté une relation commune.

La séquence liste peut bien passer pour le «degré zéro de la procédure descriptive ${ }^{12}$ »; elle est assurément une textualité minimale. Avec notre analyse du geste énumératif à l'esprit, demandons-nous désormais pourquoi, en contexte de production écrite, la liste est une méthode. Pourquoi est-elle propice à l'invention et à la préparation écrite et quels aspects du texte prépare-t-elle?

\section{Fixation}

En premier lieu, le listeur confie son matériau langagier à la rémanence du signal graphique : «Depuis l'Antiquité, de fait, l'art de la liste est un art de la mémoire [...]13. »Par sa liberté de plan et son minimalisme potentiel, la liste dispose par ailleurs à une fixation rapide des

12. Jean-Michel Adam, Les Textes : types et prototypes, Paris, Nathan, «Université», 2001, p. 81.

13. C'est Christelle Reggiani qui le rappelle ici à propos de Perec, pour qui «le recours à la liste constitue une procédure génétique usuelle, voire préférentielle» («Poétique de la liste. Inventaire et épuisement dans l'œuvre de Georges Perec», dans Liste et effet liste en littérature, op. cit., p. 506 et 507).

\section{Du geste énumératif dans la genèse des ouvres}




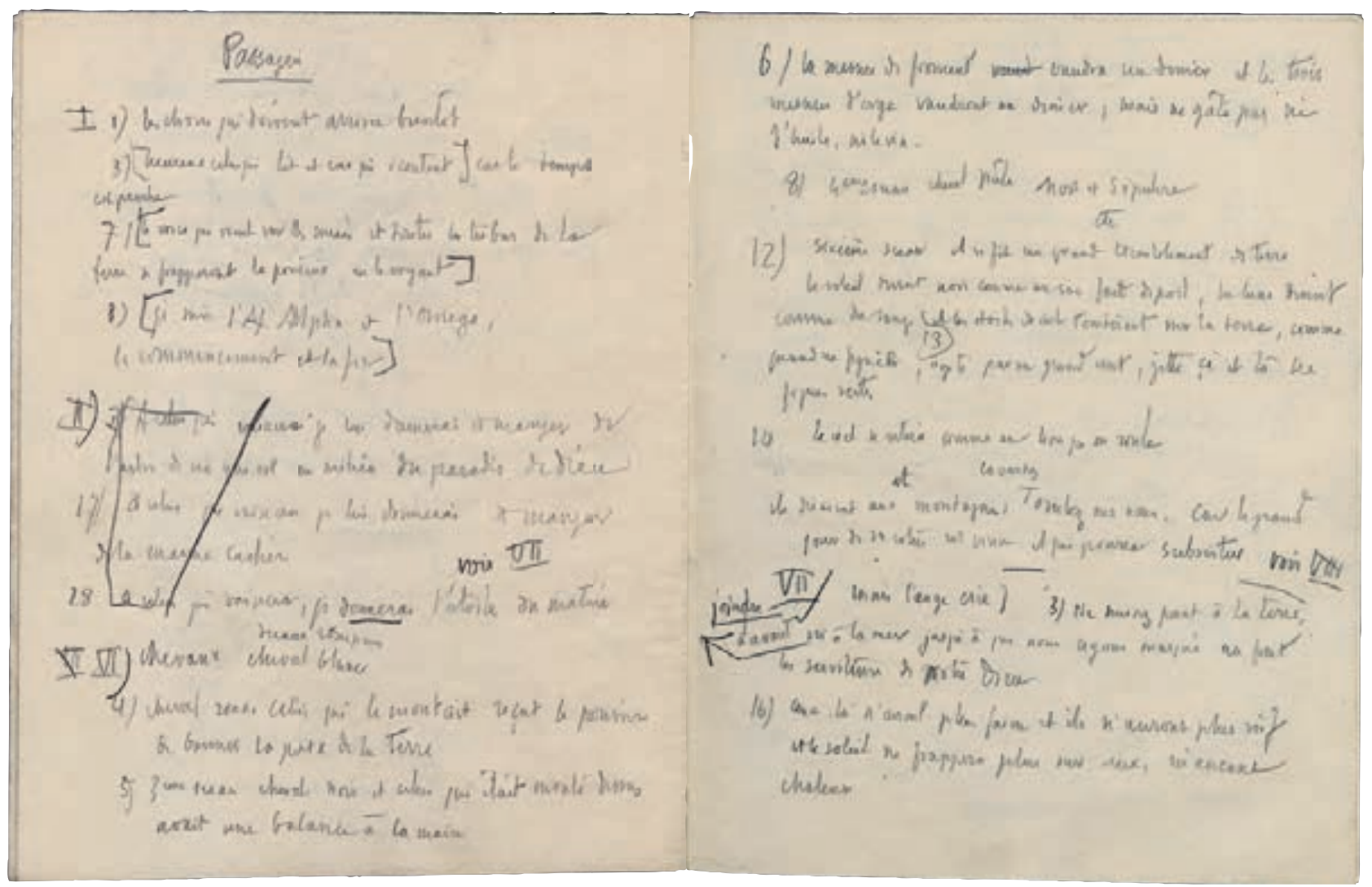

Fig. 1 : Double page du petit cahier bleu de Ramuz, présentant une liste de citations de l'Apocalypse intitulée «Passages». (C) Christophe et Laure Brossard; crédits photographiques : Bibliothèque cantonale et universitaire, Lausanne

Quelques notes prises dans le journal de Ramuz, le 30 juillet 1918, constituent le premier témoin de la genèse des Signes parmi nous. S'ensuit, en août, une ébauche des trois premiers chapitres. Arrêté par la création, avec Igor Stravinsky, d'Histoire du soldat, l'écrivain annonce dans son journal un «départ sérieux dans l'Apocalypse» en novembre. Un petit cahier bleu d'une vingtaine de pages, daté du 23 novembre, porte les traces de ce redémarrage. Le livret présente le scénario des chapitres, biffé au crayon, sans doute à mesure que la rédaction avance, et se termine par deux listes. La première, «Passages », se compose de cinq pages d'extraits de l'Apocalypse qui serviront de réserve «intertextuelle» : le futur roman, qui raconte comment un colporteur biblique parvient à faire passer une journée orageuse de 1918 pour la fin des temps, sera truffé de citations, d'allusions et d'emprunts terminologiques au livre de saint Jean.

contenus ou des expressions. Il n'y a guère d'activités d'écriture plus à même de suivre la dictée de la pensée. Du point de vue de la lecture, le texte-liste, en vertu de ou par son économie, dispose à la saisie globale. Nous aurons l'occasion d'y revenir.

La simplicité de la liste a bien sûr une contrepartie. Les relations entre les composantes listées n'étant pas explicites, celles-ci doivent être identiques, évidentes pour le scripteur ou encore non pertinentes à ce stade de la conception du texte.

La fonction de stockage et le minimalisme textuel de la liste expliquent son intérêt pour la réalisation de tâches algorithmiques; qu'on pense aux listes de courses et aux to-do-lists : elles ont bien pour fonction de préparer et de soutenir l'action en déchargeant l'activité cognitive de la mémorisation de la chaîne d'opérations nécessaires à l'accomplissement de la tâche. Mais si l'on veut prendre la mesure des bénéfices de la liste pour les tâches heuristiques, il faut mettre au jour d'autres de ses fonctions essentielles.

\section{Prédisposition}

Peut-on mettre en rapport le degré zéro de textualité qui caractérise la liste avec le moment zéro de l'écriture où on la pratique souvent? Ainsi formulée, la question appelle une première 


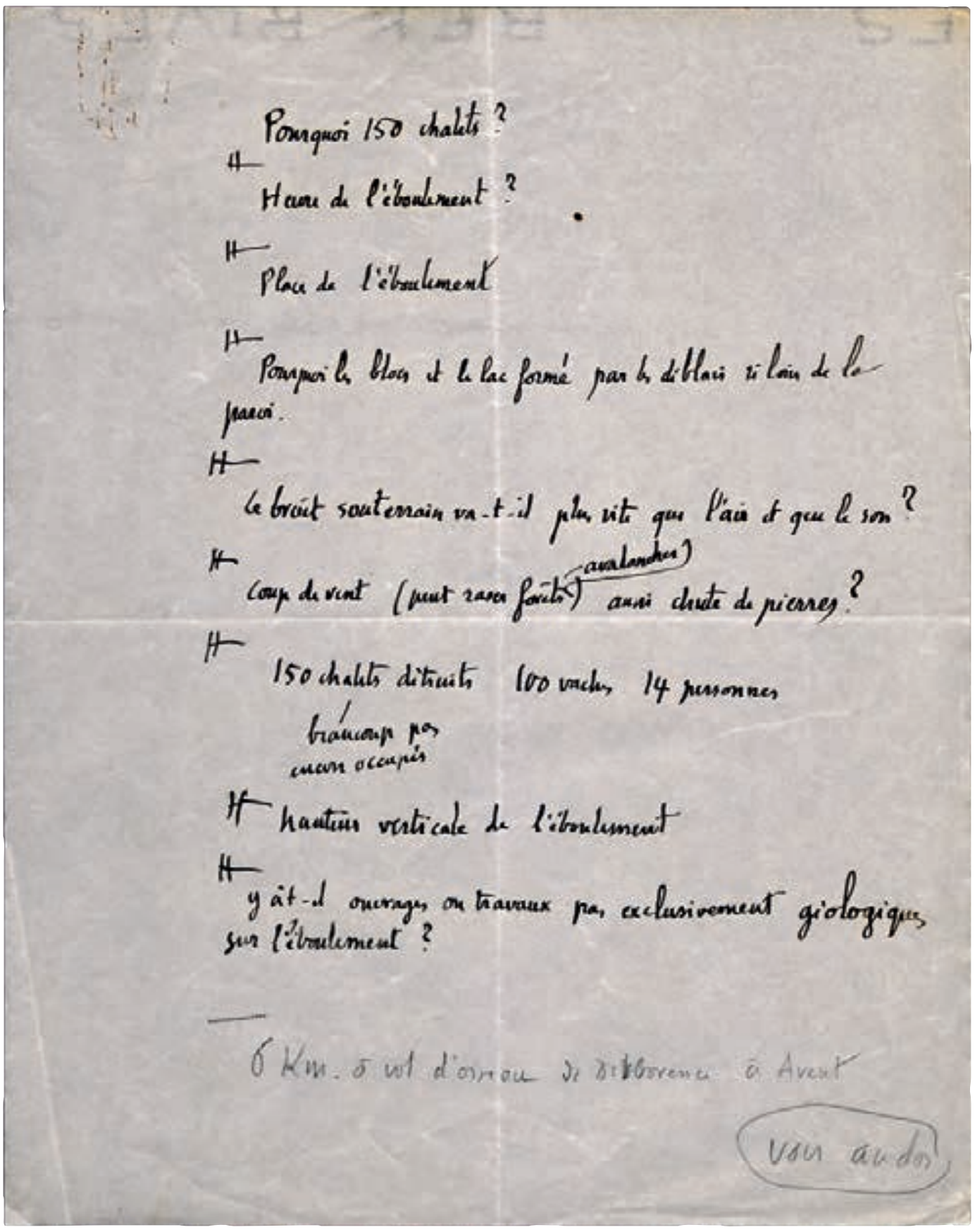

Fig. 2 : Liste de questions soulevées par la consultation du Dictionnaire géographique de la Suisse. () Christophe et Laure Brossard; crédits photographiques : Bibliothèque cantonale et universitaire, Lausanne

Le dossier génétique de Derborence s'ouvre sur trois feuillets : les deux premiers sont couverts de notes tirées du Dictionnaire géographique de la Suisse: Ramuz y cherche, le 20 juin 1933, des informations historiques concernant un célèbre éboulement près du village d'Avent et un berger qui y aurait survécu. Le document s'achève sur une liste de questions soulevées par la consultation de la source. Avant de partir dans la scénarisation de son récit, le scripteur manifeste le souci d'orienter, par cette liste, la suite des recherches documentaires ainsi que l'invention de détails factuels manquants, dans l'esprit d'une fiction historique. 
réponse. Le scripteur trouve des avantages à entrer «en douceur» dans la textualité, la liste constituant le seuil textuel minimal.

Au moment de lister, le scripteur n'a pas encore à établir l'ordre dans lequel les éléments seront organisés (dans le roman, le poème, le recueil... en cours de préparation) : il peut suivre l'ordre de sa pensée, sans se charger encore de l'ordre du texte préparé. Le processus de création est momentanément allégé de la tâche de composition et se concentre sur l'invention des ingrédients. En bref, la liste est une pratique préparatoire qui écarte la disposition, met de côté la structuration ordonnée et hiérarchisée du texte préparé. Cette lacune fait sa force.

Le caractère «pré-dispositionnel» de la liste permet par exemple à Guy de Pourtalès de lister des thèmes de son roman sans se préoccuper encore de leur agencement (p. 23); il permet à Perec de lister des mots sans $e$ (p. 119), ou à Zola, des mots d'argot selon une progression qui n'est pas contrainte par l'ordre de leur emploi futur... Si la liste apparait souvent à l'entrée de la phase scripturale de la genèse, c'est sans doute qu'en reportant la recherche de l'organisation du texte, elle permet de se focaliser totalement sur l'invention de ses composantes.

Par suite, alors que l'énumération devient liste - et le processus trace -, celle-ci trouve dans l'espace graphique un nouveau fondement à son pouvoir d'engendrement. Des unités textuelles écrites, combinées dans un geste de liberté syntagmatique, disposent le scripteur à explorer des arrangements possibles. L'œil et l'esprit associent, dans l'espace, les items jetés ensemble et mis en facteur commun par la liste; ils explorent leurs potentialités sémantiques et expressives, décèlent des ingrédients manquants qui compléteraient les relations entre items déjà énumérés et enfin découvrent de possibles mises en texte. La description donnée par Jacques Neefs des listes de La Disparition illustre idéalement cette dynamique, où la trace du processus amorcé devient la ressource de sa continuation.

On notera que dans cette dynamique, où la contiguïté des unités textuelles prépare la textualité, où l'espace nourrit le sens, ce n'est pas seulement la vision qui apporte son concours à la création, c'est aussi la main. Flèches et numérotations attestent souvent un geste de mise en ordre (fig. 3). Ce qu'illustre la présence de ces symboles graphiques, c'est le passage du geste énumératif propre à la liste à une activité de disposition, propre au plan.

Le fléchage et le raturage des listes témoignent de la proximité et de la différence de deux tâches génétiques : inventer-stocker les composantes du projet et planifier leur ordre 14 . Ce sont ainsi souvent les mêmes documents qui portent les traces de ces deux activités : la première campagne d'écriture vise la collection, l'ordonnancement est l'objectif des campagnes suivantes. D'autres fois, la mise en plan du matériel collecté par la liste opère lors du passage à un autre support, attestant ainsi plus clairement des finalités distinctes, bien qu'étroitement corrélées, des deux activités. L'énumération et la liste ne sont pas des pratiques de l'arrangement en elles-mêmes, mais elles y sont étroitement liées, comme une condition préalable. Dans l'ordre des textes préparatoires, à la liste succède régulièrement le plan.

En bref, l'activité énumérative et la liste qui en résulte fonctionnent comme une alternance de phases d'écriture et de lecture au cours desquelles le scripteur profite de la productivité du

14. Monica Zanardo cite, dans ce numéro (p. 46), une étude des manuscrits de l'éloge d'Esprit Fléchier par D'Alembert qui analyse un bel exemple de cette succession directe des gestes de mise en réserve puis d'organisation par numérotation et fléchage. 


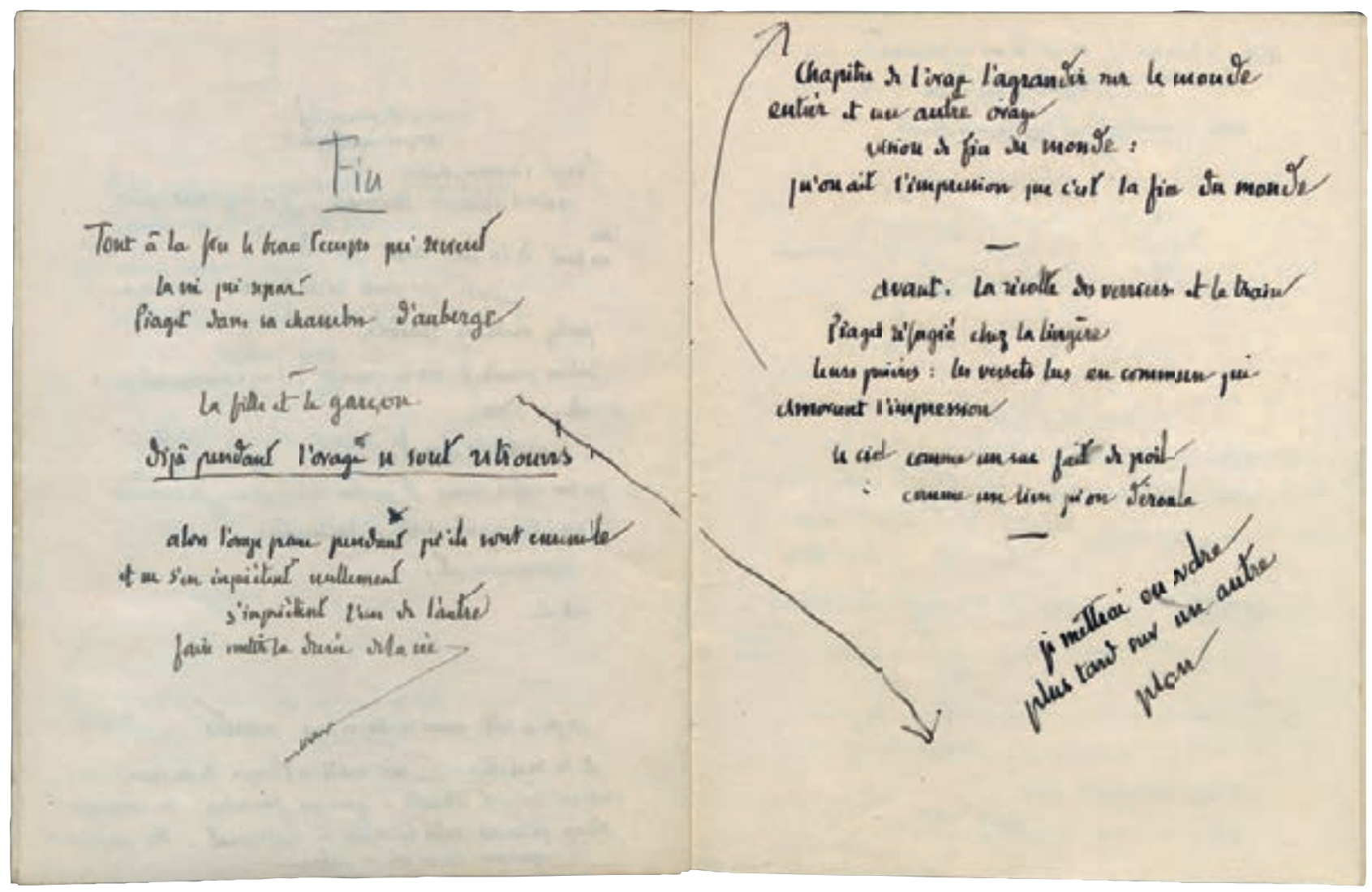

Fig. 3 : Pages tirées du cahier bleu de Ramuz, préparant la «fin» des Signes parmi nous. La logique du geste premier est énumérative : le scripteur commence par la situation finale («Tout à la fin le beau temps qui revient...»), puis il ajoute des blocs narratifs, séparés par de petits traits horizontaux. L'activité de préparation a d'abord pour objectif d'inventorier les scènes conclusives, isolées et inarticulées. L'ordre semble survenir d'autant plus facilement, au cours de l'inventaire des ingrédients, qu'il n'est pas un objectif premier : comme l'idée, venue après, d'ajouter un épisode «avant» (page de droite), les flèches venant modeler, après coup, la disposition ou encore la consigne explicite : «je mettrai en ordre plus tard sur un autre plan ».

(c) Christophe et Laure Brossard; crédits photographiques : Bibliothèque cantonale et universitaire, Lausanne

déjà énuméré pour relancer le geste énumératif, étendre le stock des unités, éprouver leurs associations et s'engager parfois déjà dans l'organisation du texte en préparation.

\section{Dérivation}

La productivité de l'activité énumérative provient enfin de la dynamique qu'elle suppose entre un mouvement synthétique et un mouvement analytique. D'une part, la liste suppose, on l'a vu, un tout, une classe, éventuellement verbalisée par un titre qui ancre le projet dans une notion ou une expression : un objet à représenter, une tâche à accomplir, un genre... La liste suppose d'autre part des items multiples, dérivés de la classe par des opérations cognitives diverses mais comportant tous avec elle une relation commune.

L'efficacité heuristique de l'opération énumérative dépend de la capacité du scripteur à décliner ou à dériver, bref à inventer des ingrédients à partir de la classe. Dans le sens inverse, la création par énumération suppose également de réunir ou de subsumer les items en une classe, bref d'inventer le tout auquel contribueraient les éléments préalables. Les carnets de notes, comme ceux de Joyce, illustrent une méthode de création partant résolument de la multiplicité des items : ils se composent de listes qui sont les ingrédients d'un texte futur, à condition qu'un principe unitaire, encore à trouver, permette le développement du paradigme en une textualité. Inventer en énumérant, c'est dégager l'unité derrière le multiple des items 


\section{Des listes génétiques pour quoi faire?}

(construire la cohérence) et dégager le multiple derrière l'unité d'une classe (construire la différence et la progression).

En résumé, la liste génétique est un détour textuel et méthodologique, un échafaudage de la création du texte final. Le coût de sa construction, relativement faible, doit se justifier néanmoins par ses bénéfices : moment de la création dévolu au stockage ou à l'invention de composantes (et non à leur organisation), durant lequel le scripteur peut suivre les informations telles qu'il les rencontre, ou ses idées «comme elles viennent» et telles que les stimule la dialectique inhérente au geste énumératif entre principe d'unité (ce qu'ont les items en commun) et principe de diversité (ce qui singularise chaque item comme ingrédient nouveau).

Sur la base de notre analyse de l'activité énumérative puis de l'analyse des intérêts de la liste en contexte de production écrite - geste libéré de l'objectif d'organisation, concentré sur la fixation ou l'invention des composantes textuelles et stimulant la découverte de composantes nouvelles et la planification -, brossons une typologie des listes génétiques. Les types proposés ne sont pas des catégories au sens logique du terme, mais correspondent à des finalités prototypiques. Pour les identifier, j' ai tenu compte des deux caractéristiques suivantes : (1) statut des items dans la liste (solutions concurrentes $v s$ ingrédients à combiner) et (2) origine du matériau listé (inventé $v s$ recopié).

Dans la description des listes génétiques quelles qu'elles soient, il importe encore de prendre en considération leur position sur deux axes : celui la chronologie de la genèse, entre le pôle des listes primitives et celui des listes tardives; et celui de l'empan concerné par le document préparatoire : de la liste locale (préparant une partie d'un texte : une scène, un dialogue, une description...) à la liste globale, préparant un texte entier.

La trentaine de listes passées en revue au moment de rédiger cette étude ne suffit assurément pas pour prétendre à un inventaire exhaustif des usages. D'autres recherches à venir viendront, espérons-le, compléter et corriger cette première proposition.

\section{Listes d'invention (des ingrédients textuels)}

Inventer par la méthode liste peut d'abord consister à jeter sur le papier, à fixer et enfin à stimuler l'invention des possibles ingrédients du texte projeté. Lorsque de telles listes sont primitives, elles visent à engendrer, dès l'entrée en écriture, la collection des composantes du texte projeté. Le geste de programmation est alors maximal, bien qu'il ne vise à préparer, rappelons-le, que le matériau textuel et non son organisation (ce sera le rôle du plan).

Les ingrédients de la liste, en contexte génétique, ont un statut instructionnel : ils réunissent des formes «à utiliser», des thèmes «à développer», des problèmes «à résoudre»15. Il se pourrait que régulièrement, ce caractère instructionnel soit explicite, comme c'est le cas de la liste par laquelle Valéry prépare «La Toupie» (citée ici par Johansson) : « $1^{\circ}[\ldots]$ Essayer de donner l'impression d'observation "scientifique"? / $2^{\circ}$ Utiliser dans les masses du récit mes notes sur sommeil et rêves. Théorie des phases» (p. 69). Il convient donc d'entendre ici

15. À propos du caractère instructionnel des documents préparatoires, voir Almuth Grésillon, «Langage de l'ébauche : parole intérieure extériorisée », Langages, $n^{\circ}$ 147, 2002, p. 19-38; et Rudolf Mahrer et Valentine Nicollier, art. cit., p. $210-211$. 
le mot ingrédient en un sens très large, intégrant des unités de l'expression (des «formes »), des contenus (des «thèmes »), mais aussi des actions dont l'effectuation doit aboutir au produit textuel visé.

Le feuillet issu des archives de Guy de Pourtalès (fig. 4) illustre un cas de liste d'invention par laquelle l'écrivain envisage un roman au stade initial de sa préparation graphique. La Symphonie lacustre est un cycle romanesque dont seul le premier roman sortira de presse, en 1937 : La Pêche miraculeuse. La page manuscrite ci-dessous appartient aux notes préparatoires du troisième et dernier volet. Comment l'écrivain genevois envisage-t-il le roman au stade initial de sa préparation graphique? À la question «comment», le lecteur attend que l'auteur y réponde et de manière formellement simple, d'autant plus si le propos est compliqué. Le document, en ouverture du dossier génétique, porte la trace, dans l'ordre, d'un essai de titre, de deux propositions de scénographie diaristique et d'une liste comportant elle-même un titre. Le premier moment de l'écriture enchaîne donc une opération d'ancrage notionnel («La Tempête apaisée», expression désignant, métaphoriquement, la logique

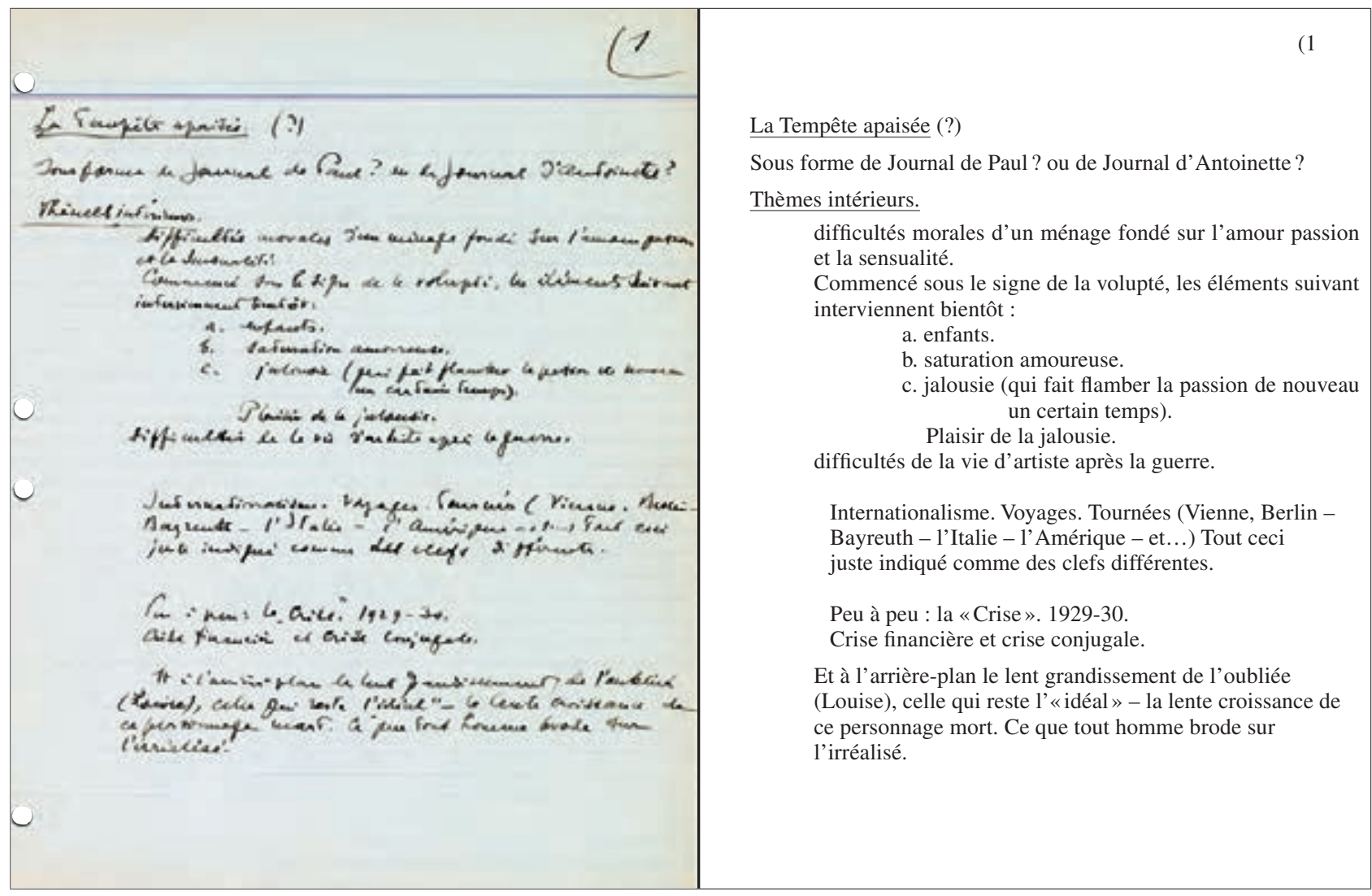

Fig. 4 : Guy de Pourtalès, notes préparatoires de «La Tempête apaisée», troisième volet de La Symphonie lacustre. Fondation Guy de Pourtalès; crédits photographiques : Centre de recherche sur les lettres romandes, université de Lausanne 
objective du texte à construire), un ancrage générique (la scène d'énonciation ou logique subjective du texte en préparation) puis une activité d'énumération, analysant les composantes du texte et explicitant leur contribution sémantique commune à la classe, en tant que «thèmes intérieurs». Le feuillet suivant comportera d'ailleurs les «Thèmes extérieurs» dessinant l'arrière-plan géographique et social au projet narratif.

Si la liste permet la liberté dans les associations et la progression des idées, on ne s'étonnera guère que, le projet étant narratif, l'énumération des «thèmes» tende à adopter une chronologie («bientôt», «peu à peu»). Ainsi, dans le premier des deux ensembles de «difficultés » prévus (les «difficultés morales d'un ménage fondé sur l'amour passion et la sensualité»), c'est en suivant des schémas actionnels internes à la classe «jeune couple passionné» que progresse localement le texte préparatoire. La réduction du projet romanesque à des thèmes ne résiste pas; la liste, en raison de l'objet qu'elle décline, tend à progresser narrativement. Les thèmes n'en sont plus, mais la capacité d'aborder le projet d'écriture sous l'angle d'une seule catégorie et de sa déclinaison a rempli son office de déclencheur scénarique.

La programmation par liste d'invention des ingrédients textuels ne saurait avoir pour effet de chasser l'arbitraire de la création, même s'il s'agit là d'un but que le scripteur peut lui assigner. Certes la mise en texte d'après liste n'avance plus librement au milieu des possibles ouverts par les langues et les discours : elle puise dans un réservoir préalable. Mais ce réservoir résulte lui-même de choix antérieurs dans l'océan du dicible. La méthode liste ne fait que reculer l'arbitraire d'un rang. Elle place un écran de nécessité entre la liberté angoissante du premier projet et le texte final.

Ce que cherche à minimiser la liste d'invention primitive, ce sont les découvertes faites au hasard de la plume et au gré des circonstances; le scripteur qui liste adopte (au moins dans un premier temps) une méthode qui ne compte pas sur les associations d'idées en phase rédactionnelle pour tracer sa voie, et même qui cherche à s'en prémunir. Pour bâtir, c'est-à-dire entrer en rédaction, il part d'un stock langagier préalablement déterminé - à l'instar de la palette du peintre ou de la gamme du musicien, pour citer des comparaisons présentes chez Valéry 16.

En somme, la méthode liste trahit une inquiétude du scripteur face à sa création dont il cherche à circonscrire, contrôler, délimiter a priori le matériau - qu'il craigne d'en manquer ou au contraire d'en avoir trop, ou qu'il redoute de collecter sa matière par accident plutôt que par nécessité. Plus fondamentalement encore, la pratique de la liste primitive suppose un imaginaire du processus créatif comme construction à partir de matières premières ou comme déduction à partir d'un ensemble d'axiomes. Pour entrer dans l'écriture par une liste, il faut d'une certaine façon souscrire à l'idée que le processus d'élaboration des textes puisse

16. Comme le montre admirablement Franz Johansson (p. 68) à propos de Valéry, et comme on peut également l'apprécier en travaillant sur le «cahier des charges » de La Vie mode d'emploi, le souci de construire préalablement la collection des ingrédients du texte préparé peut conduire au tableau matriciel. S'il s'agit d'une autre technologie scripturale, qui mérite une analyse spécifique, le tableau est bien fondé sur des listes, qu'il croise pour permettre une combinatoire, c'est-à-dire la découverte et le dénombrement des possibilités de regroupement d'unités puisées dans des ensembles déterminés. Les machines peuvent générer des tableaux à partir de listes - mais il reste à leur fournir les termes de la combinaison et les règles combinatoires. Après la liste, moment protologique, heuristique et exploratoire, le tableau matriciel est en somme un deuxième écran, parfaitement algorithmique cette fois, derrière lequel est refoulé l'arbitraire. 
admettre de dissocier la phase de collecte du matériau de la phase d'assemblage. À cet égard, les métaphores utilisées par les scripteurs pour penser leur activité (chez Ramuz, le poète est vigneron, vannier ou encore travaille dans les gravières) sont intéressantes à mettre en regard de leurs méthodes. Sans doute aussi qu'une telle dissociation des tâches (constitution d'un répertoire d'abord, planification ensuite) profite davantage à la préparation de certains genres de discours.

Face aux listes d'invention primitives, il sera particulièrement intéressant de se poser les questions suivantes.

- Le geste premier est-il celui de l'ancrage notionnel? La notion primitive est-elle au contraire déduite à partir d'un «nuage» de mots ou de séquences linguistiques déjà listées? Pour le savoir, il faudra considérer la présence ou l'absence d'un titre et également définir si ce titre précède la liste ou s'il est découvert après, comme un résultat de celle-ci.

- Par quel type de titres (notions primitives) s'ancre le projet d'écriture? S'agit-il d'un effet à produire, d'un objet à représenter... ? Le projet est-il d'emblée envisagé par une catégorie métadiscursive telle qu'un genre du discours? S'agit-il d'un pantonyme («La Tempête apaisée ») ou d'une expression plurielle («Thèmes primaires») désignant les ingrédients énumérés sans chercher à déterminer la notion qui les subsume? Ou des deux, comme chez Pourtalès.

- Enfin, par quel type d'ingrédients les textes sont-ils projetés? «De quoi» se compose un roman, un poème, une pièce de théâtre, aux yeux du scripteur, à son stade primitif? D'épisodes, de «sujets», de personnages, de mots... ? Voilà qui éclaire singulièrement la conception que le scripteur se fait de son projet et du genre de discours projeté.

Pourtalès, on l'a vu, prépare son roman en l'analysant en thèmes. Ce n'est pas tout à fait la même chose que de le concevoir à partir d'une banque de mots (sans «e»). L'analyse systématique des listes primitives permettrait d'en savoir plus sur le processus créatif des scripteurs à programme, mais elle renseignerait aussi sur la conception des genres préparés par énumération, notamment à la lumière du type des «briques» avec lesquelles le scripteur s'imagine édifier son œuvre.

\section{Listes-tempête (listes sélectives)}

Ces listes s'opposent aux précédentes par le statut qu'elles accordent aux items listés. Pensons aux brainstormings. Cette méthode de stimulation de la créativité consiste en une énumération de réponses possibles à un même problème; la tempête doit réunir plusieurs crânes, choisis pour leurs compétences différentes, et consiste à jeter un maximum de propositions en suspendant le moment de leur évaluation critique. C'est dans un moment ultérieur seulement que les solutions concurrentes proposées (concurrentes mais potentiellement combinables) sont soumises à l'évaluation et à la sélection. La suspension de l'évaluation et la confrontation des réponses les plus variées et les plus inattendues sont le gage de la créativité.

Les scripteurs produisent régulièrement une version spontanée et solitaire de cette activité lorsqu'ils réalisent des listes réunissant des solutions alternatives pour un même problème. On pense spontanément, en registre de création littéraire, aux listes de titres. Il serait intéressant d'envisager dans le détail les traces de cette activité pour considérer quel scripteur en est 
coutumier, quel est le nombre moyen de propositions réunies, quelle est l'hétérogénéité logique ou idéologique des solutions, et à quoi, enfin, elles aboutissent.

Parmi les listes d'invention, la liste-tempête est un script ou une routine méthodologique; elle prévoit d'emblée deux phases : la première de pléthore (alors qu'on ne cherche qu'une solution au problème), la seconde de sélection. Propre à ce second moment, la biffure dans la liste de solutions est une élimination. Alors que dans une liste d'ingrédients (dont les items ne sont pas des concurrents au même poste, mais des composantes à articuler ultérieurement), la biffure peut également manifester l'utilisation de l'item dans la phase ultérieure de la genèse 17 .

\section{Listes documentaires}

Les listes documentaires s'opposent aux listes d'invention (les deux précédents types) comme la réécriture s'oppose à l'écriture. La raison sans doute la plus fréquente du recours à la liste par les scripteurs tient en effet à la fonction de collecte et de stockage d'un matériau langagier alimentant le projet d'écriture : lecture d'autres œuvres aboutissant à des listes de citations (Carlos Liscano, p. 96), lecture de sources utiles pour la mise en place de l'univers à représenter (aboutissant potentiellement à des listes de lieux, de dates, d'événements...) ou encore lecture de dictionnaire aboutissant à des listes de mots utiles pour l'expression du texte préparé (rimes, vocabulaire technique...). Qu'on pense, parmi beaucoup d'autres, aux différentes listes qui parsèment le manuscrit de L'Assommoir, en particulier la longue liste de mots d'argot tirés du Dictionnaire de la langue verte d'Alfred Delvau (f ${ }^{\circ} 193-205$, consultables sur Gallica).

Le manuscrit de Gustave Roud (fig. 5) est décrit par l'archiviste comme le premier jet d'une traduction du poème «I limoni» d'Eugenio Montale. Roud liste dans la marge de droite des mots italiens pour lesquels il a cherché des traductions possibles dans un dictionnaire (comme le suggère le fait qu'il écrit dans deux cas «chercher aussi»). Par exemple : «agguantare $=$ afferrare : prendre, empoigner, saisir $»$. On notera que, dans ce cas, la liste documentaire adopte le mode sélectif de la liste-tempête, cumulant les bénéfices du stockage de connaissance lexicale et de la confrontation de solutions alternatives 18 .

Fixant de manière synthétique des matériaux textuels jugés utiles pour l'accomplissement de la tâche, la liste est donc une méthode consistant à renforcer le facteur «connaissance » de la créativité19. L'étude de ce genre de pratiques soulèvera notamment les questions suivantes : - À quelle phase de la genèse le scripteur effectue-t-il la recherche documentaire?

17. Ainsi, une liste d'ingrédients, de laquelle le scripteur ne prélèverait qu'une partie comme Joyce butinant dans ses carnets (voir la contribution de Daniel Ferrer, p. 90), dans une révision économique ou sélective de son réservoir, resterait d'une nature méthodologique différente d'une liste-tempête.

18. Monica Zanardo me signale que le dictionnaire utilisé par Roud pourrait être le Vocabolario nomenclatore illustrato de Palmiro Premoli, 1920 (à en croire la définition de «gazzarra», transcrite à l'identique). Il s'agit d'un dictionnaire analogique, qui donne, après la définition du mot vedette, la liste des synonymes ainsi que d'autres mots du même champ sémantique. L'instrument se constitue donc lui-même de listes documentaires de type lexical particulièrement favorables à la constitution de listes sélectives.

19. Pour décrire le potentiel créatif d'un individu, les modèles psychologiques contemporains s'accordent autour de modèles multivariés comprenant généralement les facteurs suivants : intelligence et connaissance, personnalité et motivation, facteurs émotionnels et facteurs environnementaux. Sur ces modèles, voir Todd Lubart, «Componential 


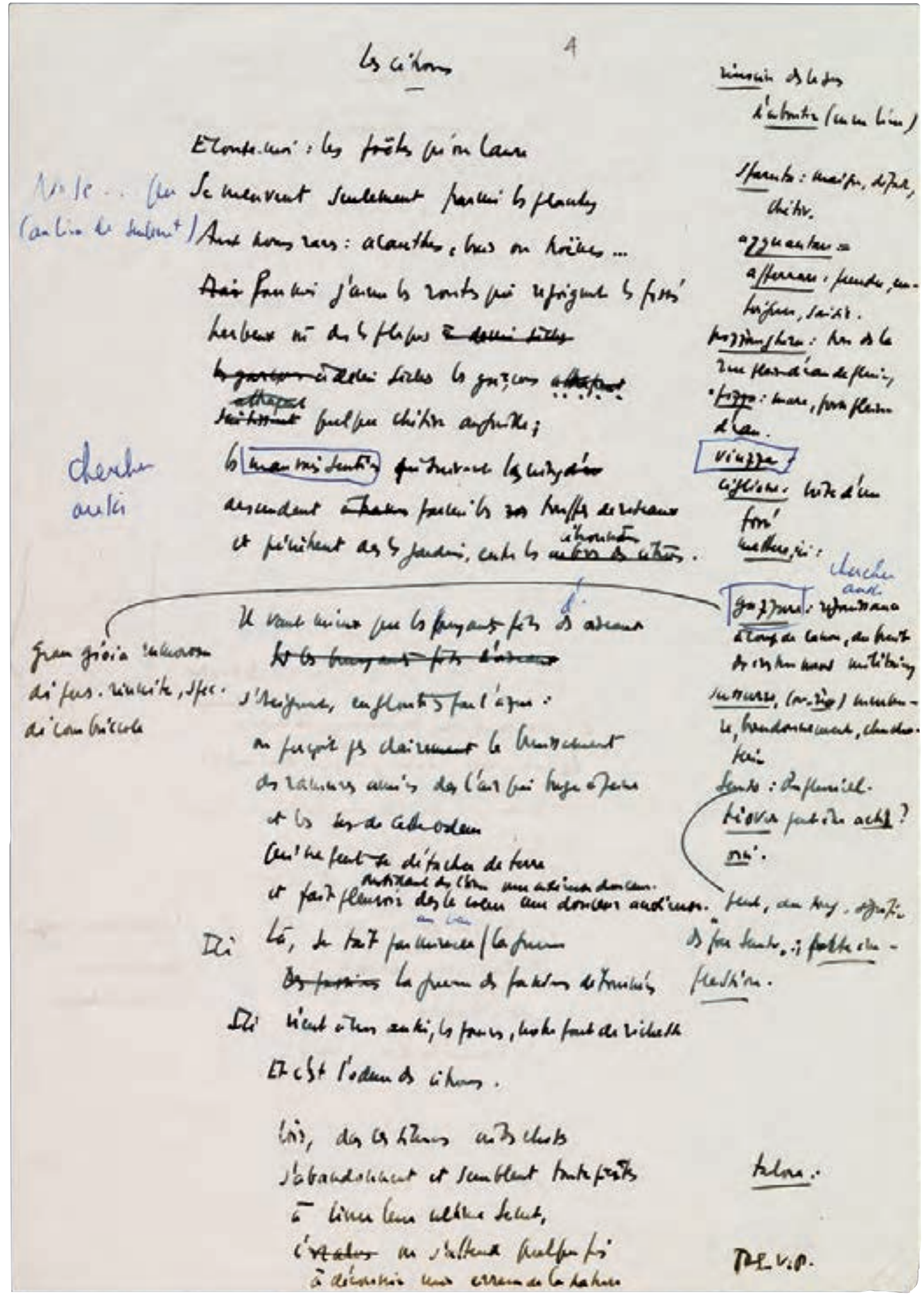

Fig. 5 : Premier brouillon d'une traduction, par Gustave Roud, du poème «I limoni» d'Eugenio Montale, à la fin des années 1950. ๑) Association des Amis de Gustave Roud; crédits photographiques : Centre de recherches sur les lettres romandes, université de Lausanne 
- De quelle nature sont les ressources langagières récoltées (énonciations, signes, signifiants ou signifiés)?

- Ou encore quel est le devenir du discours autre à mesure qu'il est incorporé dans le discours en train de se faire?

\section{Listes récapitulatives (de l'inventaire à l'invention)}

Certaines listes sont «naturellement» plus tardives; elles accompagnent le processus d'écriture et parfois succèdent carrément à une première rédaction complète du projet. L'activité énumérative a alors pour but de «faire le point» sur l'état du processus; elle consiste à réduire chaque pièce du matériau déjà écrit à une expression élémentaire (un titre, un incipit, une description identifiant la partie par son état d'avancement...) ou à un aspect du texte (personnages, lieux, mots utilisés...) et à réunir ces expressions en un même espace graphique procurant une vue synoptique et favorisant la synthèse cognitive. La liste récapitulative manifeste donc une phase d'incubation active et volontaire au cours de laquelle le scripteur ne cherche plus à produire de nouveaux matériaux, mais à renforcer le contrôle de son processus et de son produit en le relisant et le résumant.

Ce geste de recul n'est pas dénué de pouvoir d'invention. L'activité ne précède certes pas la rédaction, mais elle l'accompagne : le scripteur cherche alors à faire le point sur le matériel impliqué dans sa composition pour relancer le processus créatif. Plusieurs dossiers génétiques ramuziens attestent d'une telle routine génétique : les listes y figurent souvent non pas à l'origine du projet, mais à mi-course comme des bilans d'étape. Par exemple, l'étude du dossier génétique de Salutation paysanne conduit à observer la dynamique suivante : parmi de nombreuses listes, certaines mentionnent à la fois des «morceaux» déjà rédigés et d'autres à écrire encore; la mise en liste du déjà-produit affermit l'idée du tout et oriente l'invention des pièces «manquantes », dans une alternance entre cycle de production et cycle de réflexion synthétique sur la production ${ }^{20}$.

Le dossier d'Henri Matisse, roman (Gallimard, 1971) fournit un autre exemple de liste récapitulative. On trouve ainsi, dans des papiers de 1967, «la trame de ce roman-recueil» résumée ainsi : «Thème de madame de Senonnes, Thème de la digression de l'auteur, le personnage Douleur, les livres, la religion, la couleur - Goethe». Et Vaugeois d'expliquer : «Ces thèmes seront effectivement chacun développés dans les textes [encore] inédits mais ils reprennent et prolongent en fait les thèmes principaux de l'embryon du livre, Matisse-en-France [1943], tels qu'ils apparaissent à Aragon relisant son texte ancien'21.» La liste - d'ingrédients thématiques comme chez Pourtalès - s'inscrit donc ici entre un texte-noyau déjà publié et d'autres à produire en vue du «roman-recueil», dans un moment de récapitulation productive.

De telles listes de relance se trouvent en fait régulièrement dans le dossier génétique d'œuvres dont la structure est elle-même une liste. Comme une liste d'objets de même rang,

Models », dans Mark A. Runco \& Steven R. Pritsker (dir.), Encyclopedia of Creativity, New York, Academic Press, 1999, vol. I, p. 295-300.

20. J'ai proposé une étude de la genèse de Salutation paysanne dans les œuvres complètes de l'auteur (voir C. F. Ramuz, Nouvelles \& morceaux, t. IV, Euvres complètes, vol. VIII, Genève, Slatkine, 2007, p. 107-127).

21. Ce dossier est étudié par Dominique Vaugeois dans «Le dossier avant-textuel d'Henri Matisse, roman: perspectives sur la genèse d'une composition », Genesis, no 21, p. 95-110. 

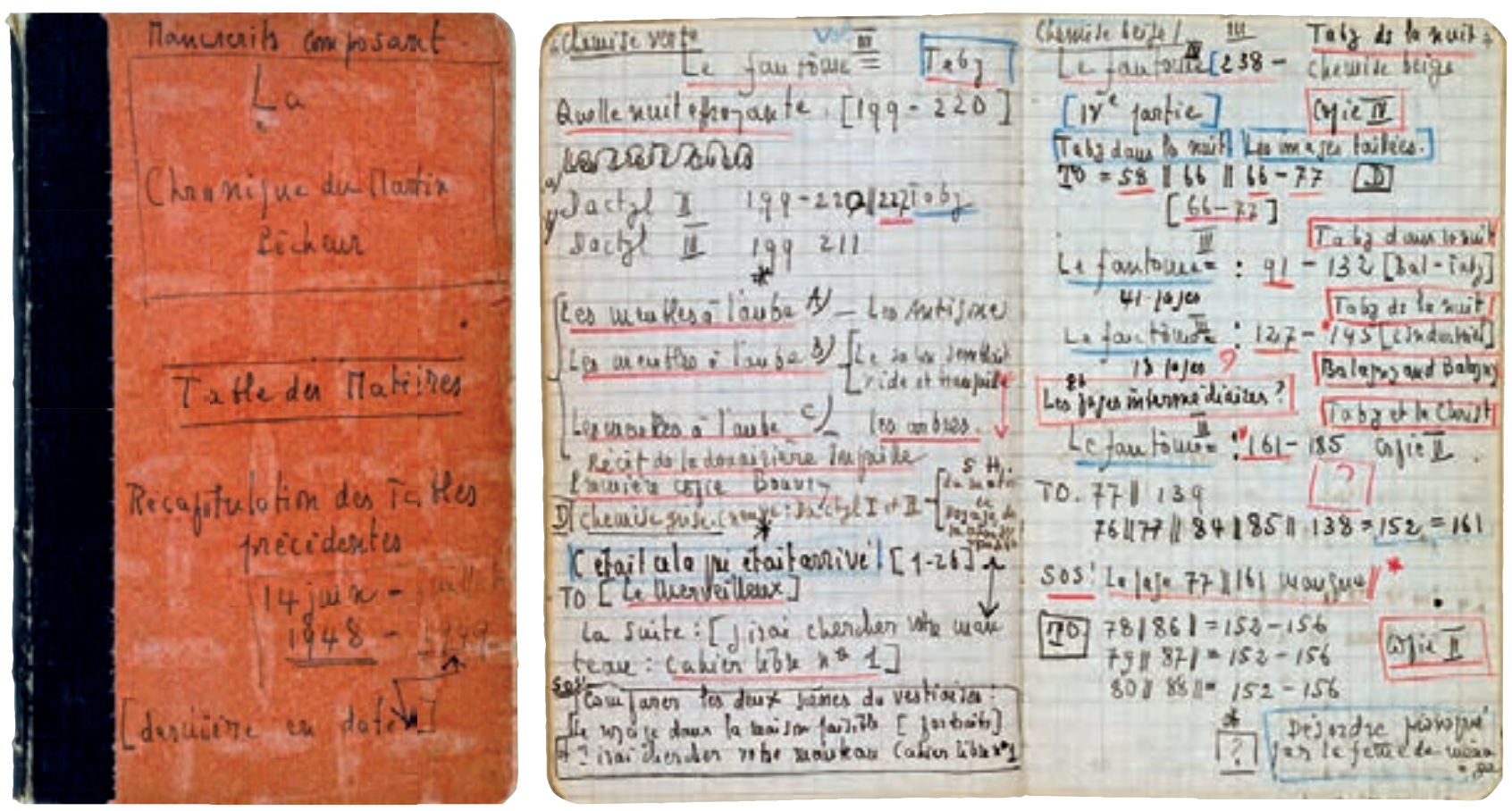

Figure 6 : Monique Saint-Hélier, «Manuscrits composant La Chronique du Martin-Pêcheur. Table des Matières », 14 juin 1948.

(C) Anne-Claude Briod; crédits photographiques : Centre de recherches sur les lettres romandes, université de Lausanne

la forme textuelle du recueil ne repose pas d'ordinaire sur une organisation englobante; il est donc plus difficile (même pour l'écrivain) d'en garder une vue d'ensemble. La liste ou le plan post-rédactionnel vient y suppléer, fournissant ainsi une vision de l'état déjà atteint, offrant à évaluer la masse de la production, sa cohérence globale et, pour le plan, son organisation en l'état.

La possibilité et la pertinence d'aborder le projet d'écriture par une liste - et donc une classe et un paradigme la déclinant - dépendent, on le constate ici, du genre du discours projeté. Si celui-ci est lui-même un genre de la liste (une encyclopédie, un herbier, un recueil...), sa préparation appelle des listes, qui sont alors des inventaires des parties du discours préparé. D'où, par exemple, le grand soin accordé par Gustave Roud à la mise en liste de fleurs dont il aimerait composer sa flore.

On rencontre ainsi un nouveau point de tangence entre plan et liste. Car les plans postrédactionnels - méthodes essentielles de l'écriture de Jean-Philippe Toussaint à en croire l'entretien accordé ici même à Gaspard Turin (p. 105) - ont une même fonction génétique (d'incubation active visant à relancer le travail de rédaction) fondée sur une force résomptive partagée avec la liste. La différence tient à la visée spécifique de ces deux activités préparatoires : le plan post-rédactionnel cherchant non seulement à récapituler le matériau déjà constitué, mais aussi à représenter l'organisation du texte préparé.

Observons enfin que le retour réflexif sur le déjà écrit peut aussi avoir pour fonction de faire de l'ordre dans les matériaux scripturaux eux-mêmes, lorsque ceux-ci prennent des proportions telles que le scripteur ne peut plus compter sur sa mémoire des états et des étapes du projet. La créativité étouffe parfois sous la masse du déjà produit. Cette situation est illustrée de manière spectaculaire par les «Tables des matières » des manuscrits composant La Chronique du MartinPêcheur de Monique Saint-Hélier (fig. 6). C'est à l'aide de carnets comme celui-ci que l'écrivaine tentait de s'y retrouver dans les milliers de pages consacrées au projet. Le Martin-Pêcheur paraît finalement chez Grasset en 1953 sous une forme réduite relativement aux premières intentions. Outre la longueur du projet romanesque envisagé, la méthode d'écriture de Saint-Hélier - de 
Fig 7 : Émile Zola, L’Assommoir, dossier préparatoire, fol. 35. Crédits photographiques : Bibliothèque nationale de France

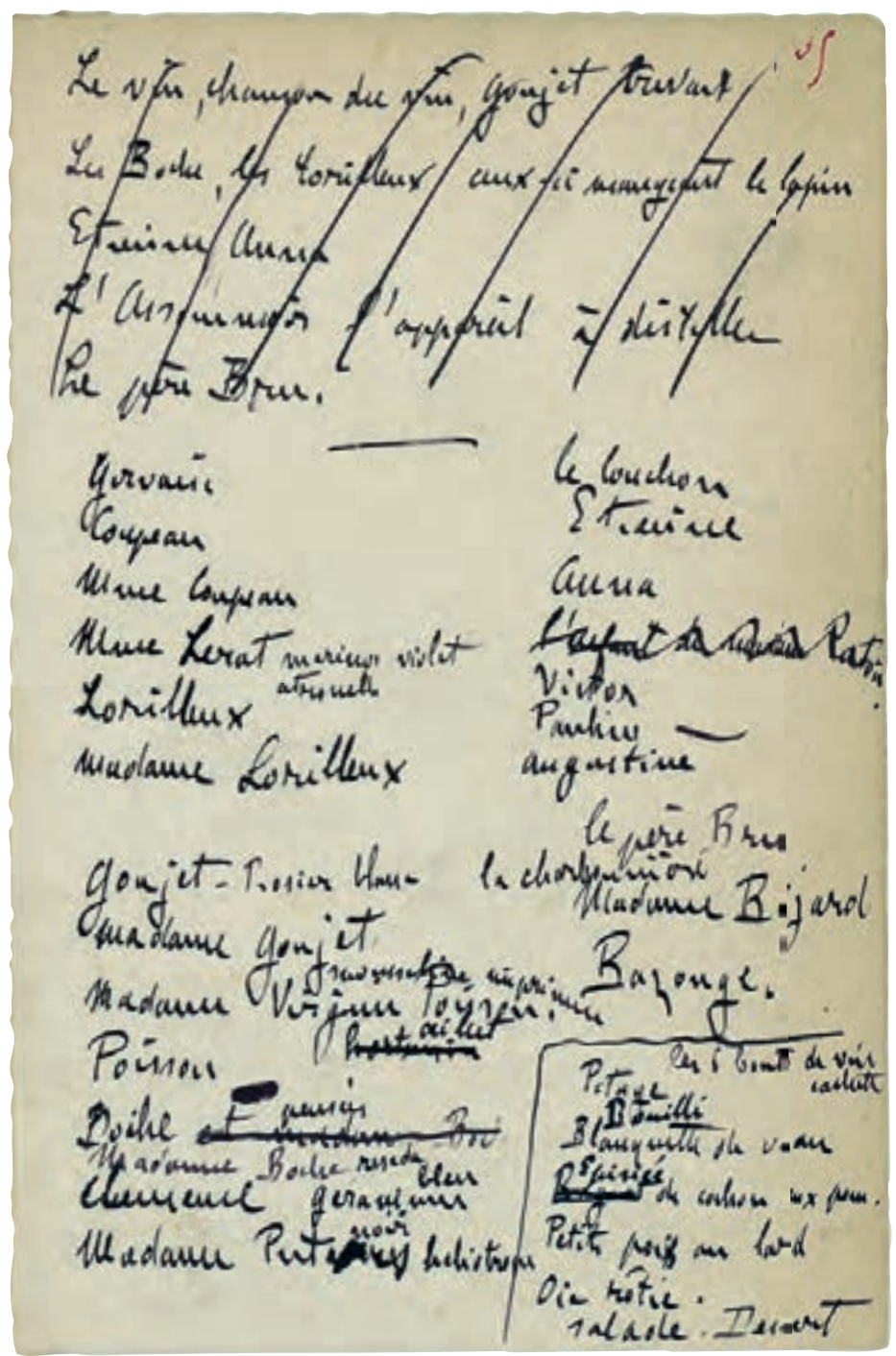

type brainstorming, consistant à écrire plusieurs versions concurrentes de la même scène pour ensuite pouvoir comparer, choisir et approfondir - explique également ses difficultés à maîtriser ce matériel. Celui-ci est dispersé sur des supports et à des endroits divers, dans des états d'achèvement variables («SOS! Comparer les deux scènes du vestiaire»), dont des parties sont parfois manquantes («SOS ! la page 77 || 161 manque») ou mélangées («Désordre provoqué par la femme de ménage »)... À l'angoisse de la page blanche répond ici la détresse du trop de pages noires. Dans les deux cas, l'inventaire offre une possible issue. La liste récapitulative rejoint alors la liste documentaire : le scripteur s'y fait documentaliste de sa propre archive.

On associera aussi à l'usage récapitulatif cette liste de personnages que dresse Zola, au milieu du manuscrit de L'Assommoir, pour préparer la rédaction de la fête de Gervaise (fig. 7). L'enjeu d'un tel document n'est plus en effet de chercher des noms; l'ensemble des items préexiste au geste énumératif. Son rôle est de délimiter, au sein d'un répertoire déjà donné, le personnel narratif de la scène en préparation. En bref, les listes récapitulatives ont pour fonction de synthétiser le déjà produit, de le constituer en base de données pour relancer de la sorte la scénarisation ou la rédaction. 


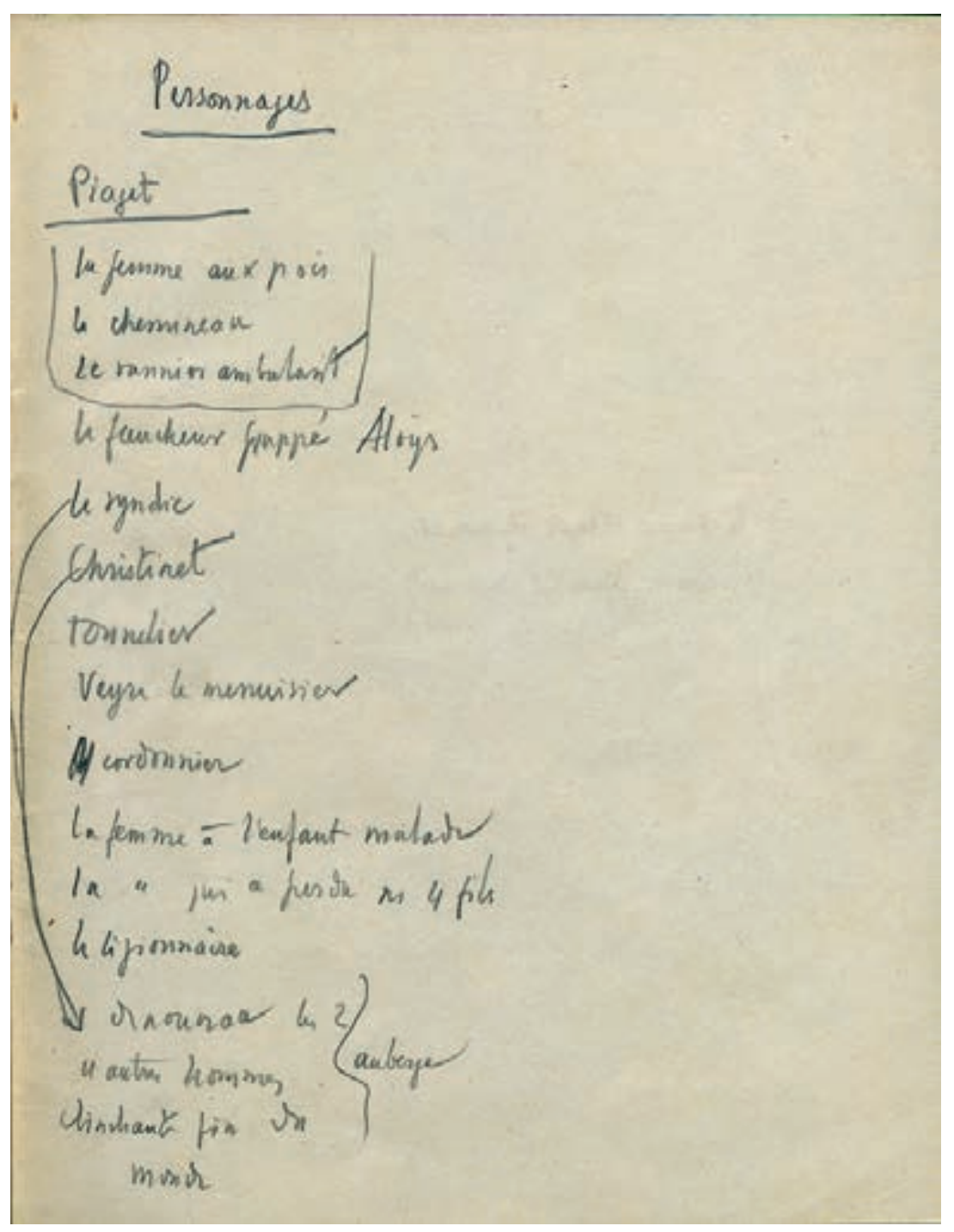

Fig. 8 : Plan schématisant la trame des Signes parmi nous.

(C) Christophe et Laure Brossard; crédits photographiques: Bibliothèque cantonale et universitaire, Lausanne

Notons pour finir que, si l'on admet de distinguer liste (geste de collection) et plan (geste de disposition) comme je propose de le faire, on ne considérera pas le document de la figure 8 comme une liste : les flèches qui pointent vers la note «de nouveau les 2 » et prescrivent le retour de deux personnages (le syndic et Christinet, dans la scène de l'auberge) indiquent bien que, ce qui est en jeu ici, ce n'est pas l'invention de la classe des personnages des Signes parmi nous, ni la mise à l'épreuve de la cohérence d'une «nomenclature» (la liste se trouve à la fin du carnet bleu, alors que tout le roman a été scénarisé); ce n'est pas non plus la constitution d'un sous-répertoire de personnages en vue de l'écriture d'une scène donnée. Ce qui est schématisé ici, c'est la progression de l'histoire rythmée par l'apparition des personnages dans le récit. Éprouvant une disposition, le document ressortit aux pratiques de la planification. On peut l'appeler liste, si l'on y tient, mais il faut alors avoir à l'esprit qu'il ne s'agit pas d'une liste telle qu'on l'a définie ici, mais d'un plan au sens où le parcours de la classe prévoit ou instruit l'ordre de la mise en texte. Si on peut parfois les confondre au niveau de leurs propriétés textuelles, énumération et planification sont deux activités cognitives et génétiques bien distinctes. 

Minimaliste,
maximaliste
Le parti pris de cette contribution était d'envisager la liste non sous l'angle strictement formel (verticalité, coordination, parataxe...), mais en combinant les propriétés formelles et praxéologiques qui définissent une pratique textuelle. Ce point de vue sur la liste, comme une séquence textuelle graphique procédant de l'activité énumérative, permet de penser celle-ci comme une méthode. Ce qu'elle est assurément lorsqu'elle est un texte qui prépare un autre texte.

La méthode liste consiste à parcourir les éléments d'une classe. En contexte génétique notamment, le parcours se fait stockage, recherche et invention : il engendre la classe. Peutêtre même la pensée de la classe et de son unité. La méthode permet au créateur de se dédier entièrement à la constitution de cette classe dans un moment, pré-organisationnel, où l'ordre de l'écriture préparatoire ne se charge pas d'épouser l'ordre de l'écrit préparé. La textualité minimale qui en résulte - sans liaison entre les parties, reposant sur une relation unique et répétée de ces parties au tout - est d'une mise en œuvre économique; l'espace graphique qui en résulte profite non seulement à la mémorisation, mais aussi à l'invention des parties manquantes et à l'exploration des relations encore indéterminées. La liste prépare ainsi le plan.

Dans le cas, le plus fascinant sans doute, de la liste d'invention primitive et globale, l'activité énumérative suppose d'entrer dans l'écriture par la dynamique d'une classe (celle à travers laquelle le scripteur projette l'œuvre à faire) et des ingrédients textuels déclinés directement de cette classe. Quelles œuvres peuvent ainsi naître d'un faisceau d'aspects ou d'une somme de parties?

En décrivant la méthode liste, dont les moyens et les fins, textuels et cognitifs, diffèrent fortement des moyens et des fins de l'œuvre préparée, nous aurons cherché à répondre à cette question simple et essentielle pour les théories de la créativité en général et la génétique textuelle en particulier : pourquoi faire une chose pour en préparer une autre? 
RUDOLF MAHRER est maître d'enseignement et de recherche à l'université de Lausanne et co-rédacteur en chef de la revue Genesis. Ses travaux s'inscrivent dans les traditions de la linguistique du texte et de la linguistique de l'énonciation et accordent une attention particulière aux différences entre oralité et écriture. Il travaille actuellement au développement de la collection numérique «Variance», consacrée à la publication des œuvres ayant été réécrites après publication (variance.ch).

rudolf.mahrer@unil.ch

\section{La méthode liste. Textualité et créativité}

Pourquoi certains scripteurs dressent-ils des listes lorsqu'ils préparent un texte? Pour répondre à cette question, l'auteur envisage la liste comme une pratique langagière graphique, caractérisable par sa forme textuelle et l'action qu'elle effectue (l'énumération). À partir d'une définition de l'énumération et d'une description linguistique de la liste, comme trace et ressource de l'action énumérative, cette contribution propose de dégager les propriétés (fixation, prédisposition, dérivation) qui rendent compte de son usage en contexte d'invention. En conclusion, à partir d'un corpus d'une trentaine de cas, s'esquisse une répartition des usages génétiques de la liste sur deux axes (liste native vs tardive, locale vs globale), puis une typologie d'usages fréquents (liste d'invention, liste sélective, liste documentaire et liste récapitulative).

Why do some writers prepare their work by drawing up lists? How do lists help their writing? To answer these questions, the author considers lists as a linguistic method, recognizable by its textual features and its activity (enumeration). Starting with a definition of the enumeration and a linguistic description of lists, as trace and resource of the enumerated activity, this paper proposes to extricate the properties (fixation, pre-disposition and derivation) that make a list useful in the context of writing. In conclusion, the numerous uses of lists in the writing process are distributed on two axes (early vs late, local vs global) and reduced at some prototypical purposes (lists for creating textual ingredients, lists for selecting, lists for documenting and lists for summarizing).

Warum erstellen manche Autoren Listen, wenn sie einen Text vorbereiten? Um diese Frage zu beantworten, betrachtet der Autor die Liste als eine grafische Sprachpraxis, die durch ihre Textform und die von ihr erfüllte Funktion (Aufzählung) gekennzeichnet ist. Ausgehend von einer Definition der Aufzählung und einer linguistischen Beschreibung der Liste, als eine Spur und Ressource der Aufzählungsfunktion, wird in diesem Beitrag vorgeschlagen, die Eigenschaften (Fixierung, Prädisposition, Ableitung) zu identifizieren, die für ihre Verwendung im Zusammenhang mit der Textproduktion verantwortlich sind. Abschließend wird aus einem Corpus von etwa 30 Fällen eine Verteilung der genetischen Verwendungen der Liste auf zwei Achsen skizziert (ursprüngliche vs. im späteren Verlauf erstellte Liste, lokal vs. global) und dann eine Typologie häufiger Verwendungen (kreative und selektive Liste, Dokumentationsliste und Zusammenfassungsliste).
¿Por qué algunos escritores elaboran listas cuando preparan un texto ? Para responder a este interrogante, el autor considera la lista como una práctica lingüística gráfica, caracterizable por su forma textual y la acción que realiza (la enumeración). A partir de una definición de la enumeración y de una descripción lingüística de la lista -como registro y fuente de la acción enumerativa-, este trabajo intenta distinguir las propiedades (fijación, predisposición, derivación) que dan cuenta de su utilización en un contexto de invención. En conclusión, a partir de un corpus de unos treinta casos, se esboza una repartición de las utilizaciones genéticas de las listas en dos ejes (lista nativa vs. tardía, local vs. global), y luego, una tipología de las utilizaciones frecuentes (lista de invención, lista selectiva, lista documental y lista recapitulativa).

$\mathbf{O}$ que leva certos escritores a elaborar listas quando preparam um texto? Respondendo a esta pergunta, o autor encara a lista como um procedimento gráfico, que caracteriza a linguagem pela sua forma textual e pela ação que executa (uma enumeração). A partir da definição deste conceito de enumeração e de uma descrição linguística da lista, como rasto e como recurso da ação enumerativa, são identificadas propriedades que ressaltam do uso da lista em contexto de invenção : fixação, predisposição, derivação. Em remate, a partir de um corpus de cerca de trinta casos esboça-se uma distribuição do emprego genético da lista segundo dois eixos (lista nativa vs tardia, lista local vs global), acompanhada de uma tipologia dos seus usos mais frequentes (lista de invenção, lista de selecção, lista documental e lista de recapitulação).

Perché alcuni scrittori compilano delle liste mentre preparano un testo ? Per rispondere a questa domanda, l'autore considera la lista come una pratica di linguaggio grafico caratterizzata dalla sua forma testuale e dall'azione effettuata (l'enumerazione). Partendo da una definizione dell'enumerazione e da una descrizione linguistica della lista come traccia e risorsa dell'azione enumerativa, l'articolo si propone di dedurre le proprietà (fissazione, predisposizione, derivazione) che rendono conto del suo utilizzo in un contesto di creazione. In conclusione, partendo da un corpus di una trentina di casi, si delinea una ripartizione degli usi genetici della lista su due assi (lista nativa vs tardiva, locale vs globale), e successivamente una tipologia di usi frequenti (lista d'invenzione, lista selettiva, lista documentaria e lista riassuntiva). 